\title{
Modeling Knowledge Transfer in a Software Maintenance Organization - An Experience Report and Critical Analysis
}

\author{
Golnaz Elahi ${ }^{1}$, Eric $\mathrm{Yu}^{2}$, and Maria Carmela Annosi ${ }^{3}$ \\ ${ }^{1}$ Department of Computer Science, University of Toronto, Canada, M5S 1A4 \\ gelahi@cs.toronto.edu \\ ${ }^{2}$ Faculty of Information, University of Toronto, Canada, M5S 3G6 \\ yu@ischool.utoronto.ca \\ ${ }^{3}$ Ericsson Software Research, Marconi SpA, Italy \\ mariacarmela.annosi@ericsson.com
}

\begin{abstract}
Modeling notations have been introduced to help understand the why behind software processes. We ask how are these techniques being used in industrial practices? The first part of this paper reports on the experiences at an industrial software organization, Ericsson Marconi SpA, in applying $i^{*}$ modeling to analyze knowledge transfer effectiveness for software maintenance. The modeling was done in-house without consultation with the $i^{*}$ research community. In the second part of the paper, university researchers analyze the modeling experience in that organization, drawing a comparison with the usage of $i^{*}$ typically envisaged by the research community. We found that the modeling approach used at the industry site employed smaller and simplified models, but were effective for highlighting key issues for the organization and communication. From the case study, we draw some conclusions for the future development of the $\mathrm{i}^{*}$ modeling approach.
\end{abstract}

\section{Introduction}

Modeling notations have been introduced to help understand the why behind software processes [1. To design effective Enterprise Modeling (EM) languages and methods, it is of importance to study how practitioners apply the methods developed by research communities in real-world practices, what are the properties of the enterprise models developed in practice, and how the EM language and method developed by researchers were understood in practice. While practitioners focus on applying the techniques for practical purposes, results of studies on adoption of EM techniques by practitioners would provide directions for future EM research toward practical proposals for modeling and analysis approaches.

This paper aims to analyze the properties of the enterprise models developed in a real-world instance. To have a basis for comparison, an alternate set of models of the same organization and problem was developed according to the researchers anticipated usage of the modeling technique. We study and compare the size, complexity, understandability, modeling style, appropriate and full use 
of the modeling notation capabilities, and deviations from the syntax of the modeling notation for the two sets of models.

The first part of this paper reports the experience of applying an agent- and goal-oriented approach to EM used in the Ericsson Marconi SpA software maintenance organization to model and analyze a number of Knowledge Management (KM) strategies applied in the maintenance team. The KM strategies applied in that organization involves restructuring roles, positions, and their activities within the enterprise. The analysts at Ericsson Marconi SpA modeled and analyzed the KM strategies and policies by using the $\mathrm{i}^{*}[2]$ agent- and goal-oriented approach. In this experience, $i^{*}$ modeling technique was used by the in-house analysts at Ericsson without consultation with the $i^{*}$ research community. The $i^{*}$ modeling and analysis were used to help the team members understand and explain the significance and impact of the KM policies they applied.

In the second part of this paper, the models developed at Ericsson Marconi SpA are analyzed to draw conclusions about the use and adoption of EM in practice. In this analysis, researchers develop a new set of models based on their understanding of the KM strategies and organizational settings at Ericsson Marconi SpA. The new set of models developed by researchers is compared with the modeling and analysis approach employed at Ericsson Marconi SpA.

\section{Case Study Background}

This section explains the organization context at Ericsson Marconi SpA and the reasons, process, and outcomes of applying i* modeling EM at that organization. The Ericsson Corporation applies benchmarking to compare its local design centers which are spread in many countries worldwide. Performance in maintaining the software products under the responsibility of a local design center is often the way to judge the effectiveness and the efficiency of that center. At the Ericsson Marconi SpA local center, a Knowledge Management (KM) project was launched to effectively manage the maintenance activities. The KM efforts involved applying organizational strategies and structures within the maintenance team which are described in more detail in section 3 .

The project leader of the KM initiative needed to describe the maintenance processes and explain how and why the KM strategies for knowledge transfer were successful in the maintenance team. Motivated by the desire and need to explain and promote successful KM practices to other groups, the KM project leader and process owner searched for a modeling method suitable for the intended purpose in the EM and process modeling literature. The project leader believed that $i^{*}$ was the only modeling notation that can express and visualize concepts like social interactions and knowledge transfer. She chose the $i^{*}$ modeling framework, while her $i^{*}$ modeling skills and expertise were limited to her own studies in the literature. The $i^{*}$ notation was chosen because she found $i^{*}$ modeling simple, easy to learn, and adaptable in industrial contexts.

The $\mathrm{i}^{*}$ modeling notation provides the means to express goals of actors and tasks and resources needed to achieve the goals. The models provide a basis 
for evaluating different degrees of goal satisfaction among different stakeholders. By making the relations between stakeholders' goals and knowledge transfer instruments explicit, the models can demonstrate how and why the knowledge instruments work or fail. These properties of the $\mathrm{i}^{*}$ modeling approach provides a suitable basis for modeling enterprise strategic structure and role dependencies.

\section{Enterprise Modeling Experience at Ericsson Marconi SpA}

This section describes the KM needs and strategies and the enterprise models developed to analyze and explain the KM strategies at Ericsson Marconi SpA. The experience of EM is described based on the internal reports provided by one of the paper's author at Ericsson Marconi SpA, who was the project leader and process owner that produced the $\mathrm{i}^{*}$ models, later consultations with that author, and a short workshop paper analyzing the $\mathrm{i}^{*}$ models [3].

The modeling process started with identification of roles, positions, and their dependencies and goals in the Ericsson maintenance team. As the modeling progressed, the model became complex. It was realized that putting all actors and processes into one model was useless, since no conclusion could be deducted from such a complex model. Therefore, an slicing approach was adopted for breaking the maintenance processes into smaller models based on the actors' goals.

The KM strategies and structures that were applied in the maintenance team target three main goals: 1) Expanding individuals' tacit knowledge by focusing on knowledge creation strategies by individuals; 2) Expanding team tacit knowledge by focusing on the social relationships among members of the maintenance organization; 3) Enabling knowledge sharing by applying KM strategies and structures to the knowledge environment.

\subsection{Expanding Individuals' Tacit Knowledge: Knowledge Creation Strategies}

Individual technical skills and knowledge about the product and system are critical to the maintenance activities. To increase the individual tacit knowledge and reduce the faults' handling costs, some KM strategies were applied in the maintenance team. Half of the maintenance team was put into a core group, which consisted of ten individuals. This group was the focal point of the maintenance team to assure knowledge sharing within cross-functional teams.

The core members need to play two roles: one as a Maintainer member and one as a Subsystem Responsible, which is a member of development project team and needs to Attend Product Committee (PC) inspection. In this way, the variety of correlated activities was guaranteed for the core members. Fig. 1 was developed to describe the activities of the subsystem responsible as an i* model: 1) To attend to the $\mathrm{PC}$ reviews as a permanent member in order to guarantee the correct evolution of products; 2) To update the subsystem documents at every project development release. 


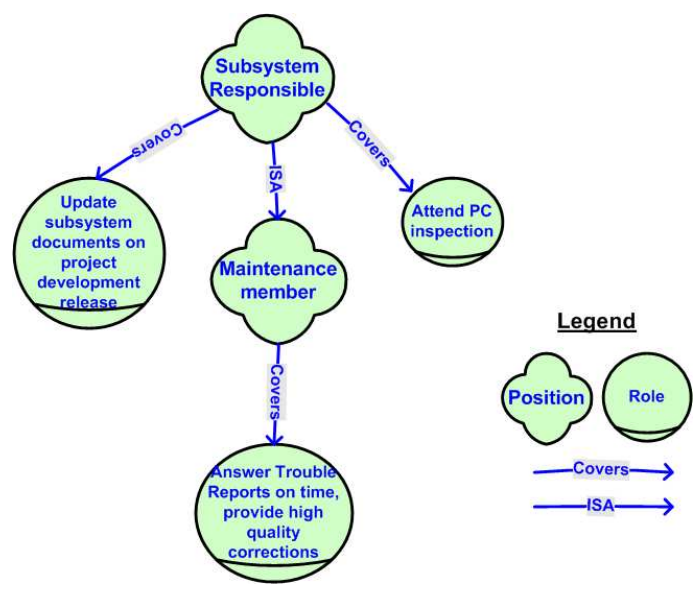

Fig. 1. Roles that belong to the subsystem responsible position. (The legends are added to the original models obtained from Ericsson.

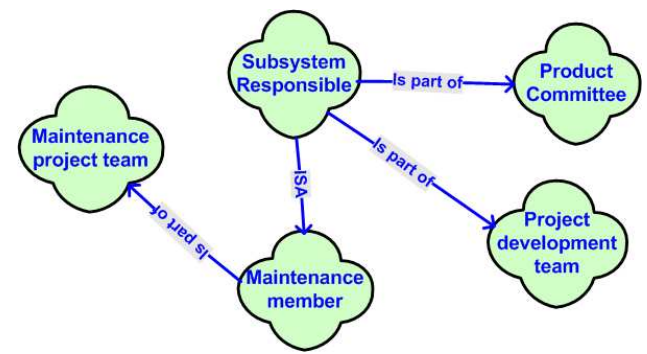

Fig. 2. Relationship between organizational positions

Ensuring Knowledge Transfer among Different Team Positions and Roles. The model in Fig. 2 was developed to describe that the subsystem responsible interacts with different organizational positions by being member of multiple teams. When the core members of the maintenance team work as a maintainer, they gain field experience by facing problems that have been discovered by the internal and external customers, solving faults, and proposing effective solutions at the product level. The maintainer as a subsystem responsible, can gain knowledge at the product level. Then later they have the chance to re-acquaint this knowledge during the evolution of the product under maintenance.

Subsystem responsibles collaborate in the feasibility phase of development project by reviewing all documents under the PC inspection. In this way, the combination of these two roles that the core members play raise the quality of the experience they gain. On the other hand, the PC also takes advantages from the specific background knowledge of the subsystem provided by the subsystem responsibles, which in turn, raises, the level of competence for the inspections. 


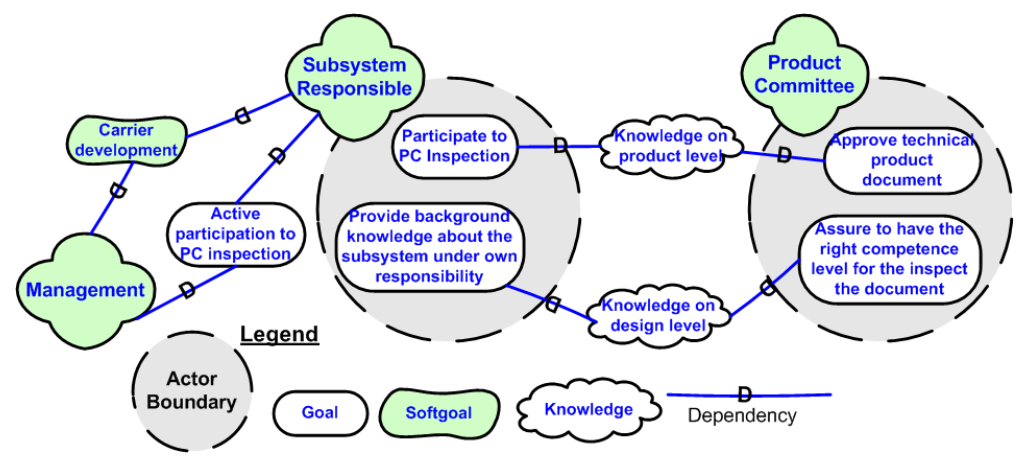

Fig. 3. Knowledge transfer from Product Committee members to Subsystem Responsible at the product level

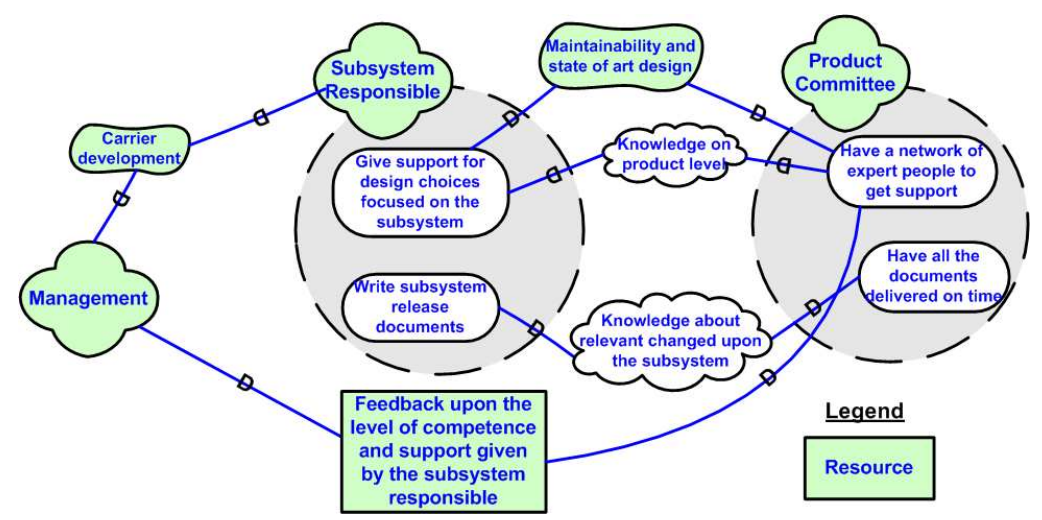

Fig. 4. Knowledge transfer from the project development team to the subsystem responsible at the design level by attending $\mathrm{PC}$ meetings

This requires that subsystem responsibles attend the $\mathrm{PC}$ for the inspection. In this way the PC inspection becomes an instrument for having an effective knowledge transfer, also in addition to the knowledge transfer by the core maintenance member.

The model in Fig. 3 was developed to illustrate the knowledge dependencies discussed. The Subsystem Responsibles are encouraged by the organization to attend the PC inspections. In addition, the Management applies some enforcement mechanism, in which the performance of subsystem responsibles is measured and evaluated by the number of attendances at PC inspection. The model in Fig. 4 was developed at Ericsson to illustrate how the new KM structure helps Subsystem Responsibles to gain knowledge. Since the Subsystem Responsibles are engaged in system development, they need to update all the subsystem release documents to record changes from the last release. To gain knowledge of product at the design level, the organization forces the maintainers to attend PC inspections for all development documents, write the subsystem release 


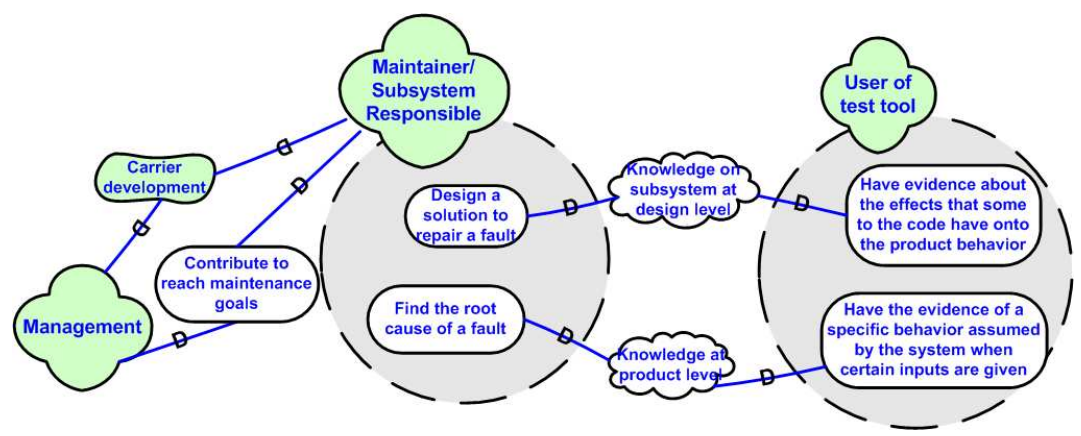

Fig. 5. Gaining knowledge through using the testing tools

documents, and communicate with the development team. The organizational interface between the Subsystem Responsibles and the Project development team is a way to guarantee an effective knowledge transfer at the design level to the Subsystem Responsibles. The model in Fig. 4, developed at Ericsson implicitly illustrates the enforcement mechanism that the management applied to ensure performance of the subsystem responsibles.

Gaining Knowledge Through Use of Testing Tools. The model in Fig. 5] was developed to illustrate how testing tools that simulate the behavior of the system are another source of knowledge for maintainers. Such tools are essentially used for test purposes by the testers. In the maintenance team, those tools are useful means to help maintainers analyze the faults individually. Knowledge creation starts when the maintainers need to simulate a fault. For this purpose, the maintainer launches the testing tools and configures it with the scenario described in the trouble report.

This requires background knowledge about similar faults, testing tools, and product at the system level. Without knowledge at the system level, simulating the fault would be burdened by a sequence of trial attempts without considering any rationale for the fault. Using testing tools, maintainers learn about the effects of the adopted solution on the whole system.

\subsection{Expanding Team Tacit Knowledge: Social Relationships Structures}

To increase the collaborations, facilitate sharing the experiences among the maintenance team members, and build mutual trust among them, the organization provided means for formal face to face communication among team members. The formal meetings that were set to transfer knowledge include desk checking the solution that fixes the fault reported in the trouble report. The model in Fig. 6 was developed to explain why and how knowledge of team members is expanded by formal desk check of the solution. The desk check is a mandatory step before delivering the corrections and is performed by two maintainers which 


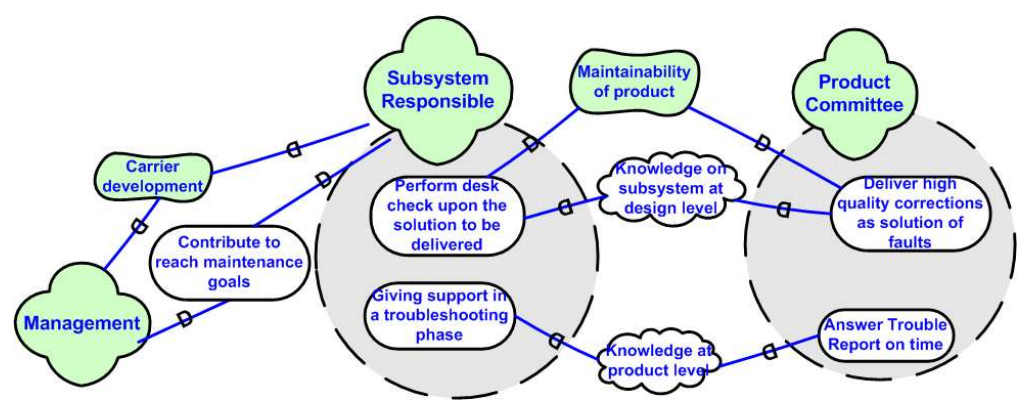

Fig. 6. Expanding knowledge of team members by formal desk check

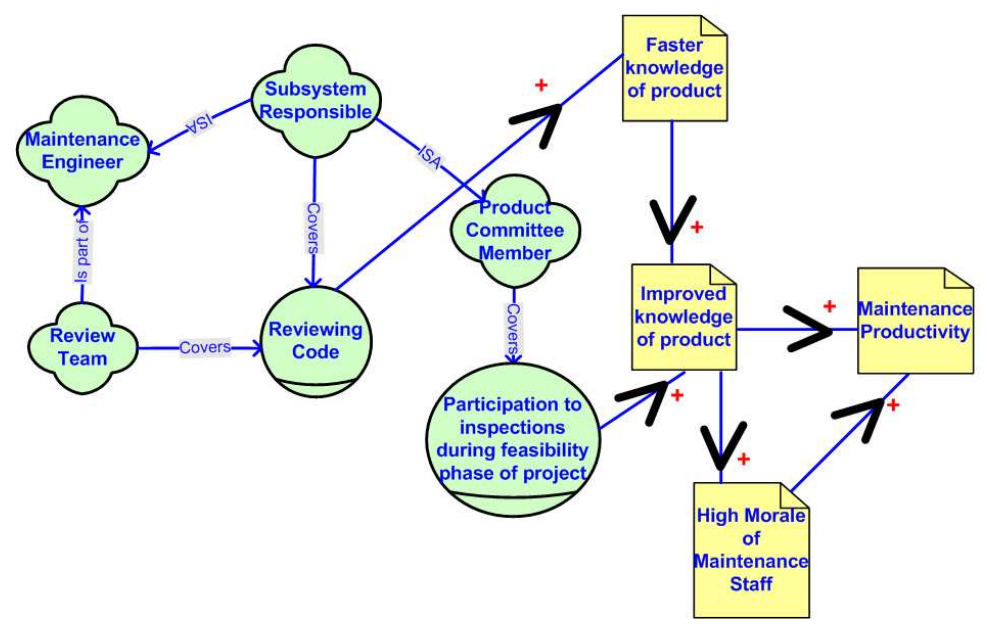

Fig. 7. Social relationships between actors within the maintenance team and the impacts of the relationships on goals of different actors. (The modeler employs a rectangle to model the softgoals, syntactically deviating from $i^{*}$ notation.)

did not fix the trouble. This check aims to find the possible faults of the solution adopted by the other maintainers.

Fig. [7 was developed to explain the social relationships between actors within maintenance team and the impacts of the relationships on goals of various roles and positions. The strong team spirit enables the interactions among the maintenance team members, and this greatly facilitates mentoring purposes. In this way, the team members feel they work more effectively and with higher level of communication.

\subsection{Enabling Knowledge Sharing: Strategies for Knowledge Environment}

Although a number of knowledge environment strategies were applied to the team environment, the KM project leader and analyst did not develop $i^{*}$ models 
for expressing the impact of physical facilities and environmental changes that facilitate knowledge sharing. Those strategies aimed to enable knowledge sharing in the organizational environment of the maintenance team. For example, the maintenance team is located within an open space consisting of several cubes, separated from each other by short walls. Team member that are doing similar types of activities are located in the same room. This helps them to have the opportunity to share their experiences.

The enterprise models developed at Ericsson express the relationships between various roles and positions in the maintenance team, the goals of roles and positions, their dependencies, and knowledge acquisitions dependencies. Utilizing the $i^{*}$ modeling and analysis technique helped the KM initiative to systematically represent, capture, and analyze the strategic organizational relationships relevant to knowledge transfer. By capturing and analyzing such strategic relationships, it is possible to make visible the reasons why newly adopted strategies and structures improved knowledge transfer.

\section{Critical Analysis of the Ericsson Modeling Experience}

This section gives a critical analysis of the modeling experience at Ericsson, drawing a comparison with the usage of $i^{*}$ typically envisaged by the research community.

\subsection{Characteristics of the Enterprise Models Developed at Ericsson}

The Strategic Dependency (SD) models developed at Ericsson explain the positions and roles at the organization. These models were used to explain additional responsibilities of the core members of the maintenance team. Strategic Rationale (SR) models refines the goal and knowledge dependencies between roles and positions. These models were used to illustrate the motivations behind the collaboration amongst different roles and positions, including knowledge transfer motivations and mechanisms.

The models developed at Ericsson involved adoption of the $i^{*}$ modeling techniques to suit the practitioners' needs. As a result, some models deviate from the $i^{*}$ syntax and modeling style laid out by the researchers University of Toronto (U of T) [2] and [4. The source for the models was the first hand experience of the modeler and the she was the key users of developed models. Reviewing and analyzing these models, we observed some common properties:

1. The models developed at Ericsson focus on one goal of each actor and a small fragment of the enterprise.

2. Each model focuses on one strategy and its benefit. In this way, each model communicates one single message.

3. The models employ a subset of the $i^{*}$ modeling elements and do not use the full range of the $i^{*}$ notation capabilities. For example, very few contribution links are used in the models, tasks are not included, and resources and softgoals are rarely employed. The most frequently used elements are roles, positions, goals, and dependencies. 
4. The models have several deviations from the $i^{*}$ syntax developed at $U$ of T. However, the models communicate the intuitions of the context, and the enterprise structure, people's activities, and goals are semantically understandable.

5. Although the syntax of the $\mathrm{i}^{*}$ notation is not accurately followed, the intuitions behind the $i^{*}$ approach for enterprise modeling is understood by the developers of the models. The $i^{*}$ models illustrate the distributed intentionality in the maintenance team and their dependencies.

6. Wherever the developer needed to express a concept that the $\mathrm{i}^{*}$ does not express or the developer did not know how to express by using the $\mathrm{i}^{*}$ modeling constructs, the developer used an improvised notation.

7. The models are not detailed and extensive. Goals are not refined into tasks and other goals using decomposition and means-end relationships. In this way, only the main important intuitions of a KM strategy are expressed in the models and details are omitted.

\subsection{Developing an Alternate Set of Models}

In order to further analyze the Ericsson experience, the $i^{*}$ researchers involved in this study developed an alternate set of $i^{*}$ models using the conventional $i^{*}$ modeling syntax which the researchers at $\mathrm{U}$ of $\mathrm{T}$ developed. The models were developed based on the description that accompanied the models provided by the Ericsson analyst. We compare the models developed by researchers with the models used at Ericsson for practical purposes, to draw conclusions about realworld application of $i^{*}$ modeling as an specific goal-oriented EM technique. Such conclusions help toward improving the practical aspects of the $i^{*}$ Framework, and EM in general. For example, in a previous work, deviations from the $i^{*}$ syntax were studied in [5]. In that study, based on the identified common deviations, suggestions for improving the usability and effectiveness of the $i^{*}$ syntax were proposed. In addition, analysis and comparison of two sets of model provide initial intuitions about how models, modeling process, and model-based analysis are adopted in real-world practices of EM.

Fig. 1 shows the model developed at Ericsson Marconi SpA to explain the roles that a Subsystem Responsible plays. This model expresses that a Subsystem Responsible is also a Maintenance member, which describes the strategy of double roles for some members. In this model, the title of roles that the positions play express the tasks and activities that the positions are responsible for. The alternate approach to model this strategy is to structure the responsibilities of a position as tasks and goals of the roles covered by the position. Fig. 8 depicts the new model of the same positions and roles based on the alternate approach used by the researchers.

Fig. 9 gives the new model corresponding the model in Fig. 3. The SR model in Fig. 3 presented the goal and knowledge dependencies among the introduced roles and positions. The model in Fig. 9] shows why and where knowledge of products or subsystems are required and how the roles can obtain this knowledge. For example, the new model captures why the Product Committee needs 


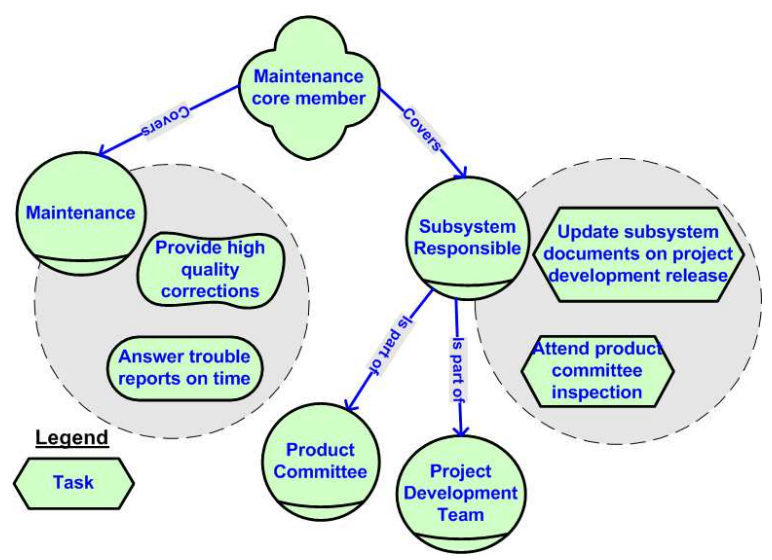

Fig. 8. A refinement of the model presented in Fig. 1 1 and Fig. 2 This model introduces special position called core member of maintenance team that covers two roles.

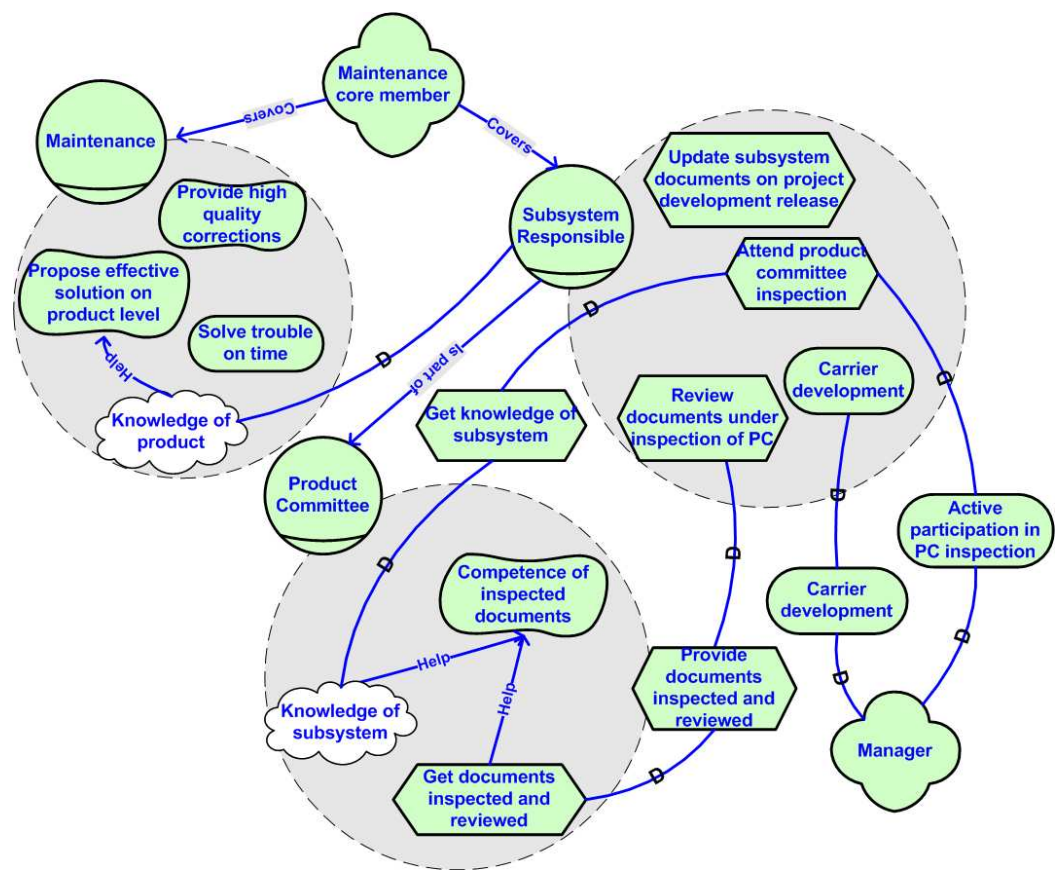

Fig. 9. A new model of knowledge transfer at the product level from Product Committee members to Subsystem Responsible. The model corresponds to the model in Fig. 3

the Knowledge of subsystem in addition to how Product Committee can obtain it. The model is incomplete, since some elements such as Provide high quality corrections are not related to other elements. The incomplete parts of the models 


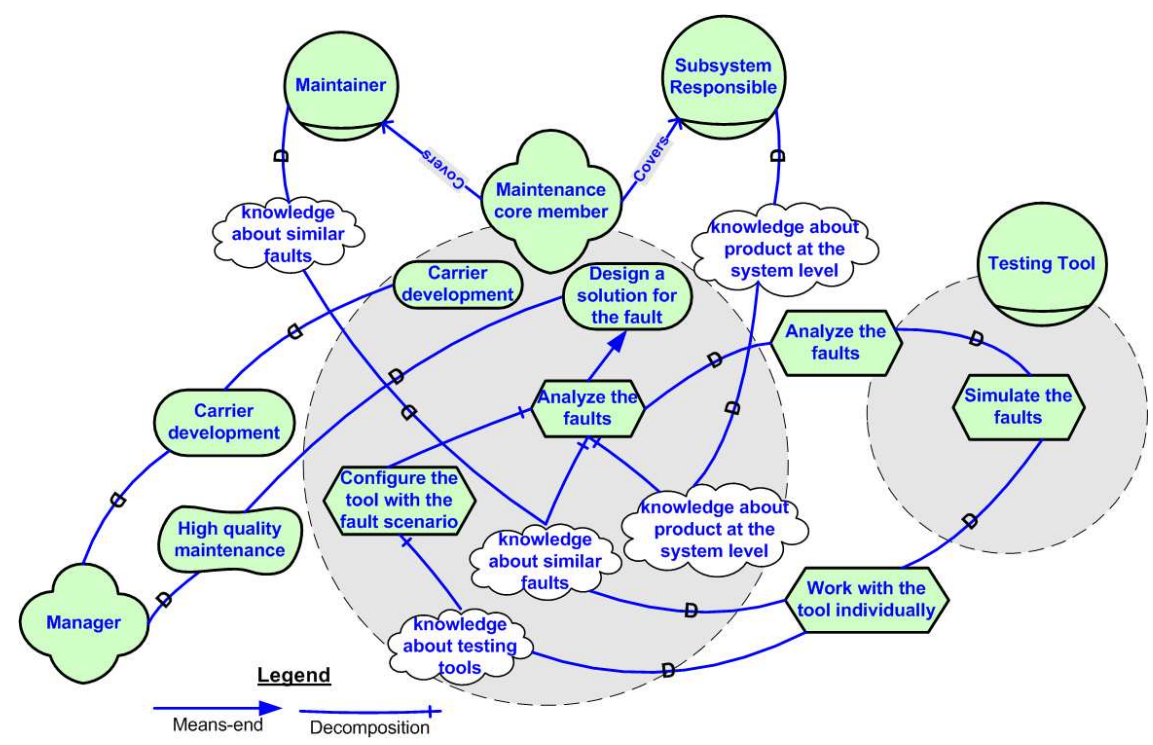

Fig. 10. A new model of knowledge transfer from Product Committee members to subsystem responsible at the product level. The model corresponds to the model in Fig. 5

are due to the lack of description in the text accompanying the models. Due to space restriction, the new refined models for Fig. 4 and Fig. 6 are omitted.

Fig. 5 shows how maintainers benefit from using the testing tool and what kind of knowledge and experience they can gain. We explain the benefits of using the testing tool in a new model developed by the researchers, shown in Fig. 10 . In this model, the required knowledge such as knowledge about similar faults and knowledge about test tools is modeled as a decomposition of the Design a solution for the fault task. In this way, the model expresses why that piece of knowledge is required. Similar to the model in Fig. 5 the knowledge elements are linked to tasks and goals of other actors by dependency links. For example, the Maintenance core member's main goal is Design a solution for the fault. To achieve this goal, the Maintenance core member can analyze the faults by simulating them on the testing tool, and in order to do this, the maintainer needs to have knowledge about test tools. To gain this piece of knowledge the maintainer depends on using the Testing Tool.

The model in Fig. 7 was developed at Ericsson to show the strategic relationship in the maintenance team and the impacts of the relationships on goals such as Maintenance productivity. This model makes use of a new symbol to represent quality goals. The $\mathrm{i}^{*}$ syntax does not allow linking actors to softgoals by contribution links. In addition, instead of actors, goals or tasks of the actors affect other goals. Therefore, we developed a new model, shown in Fig. 11, to express the intuitions of the model in Fig. 7, avoiding the syntactical deviations. 


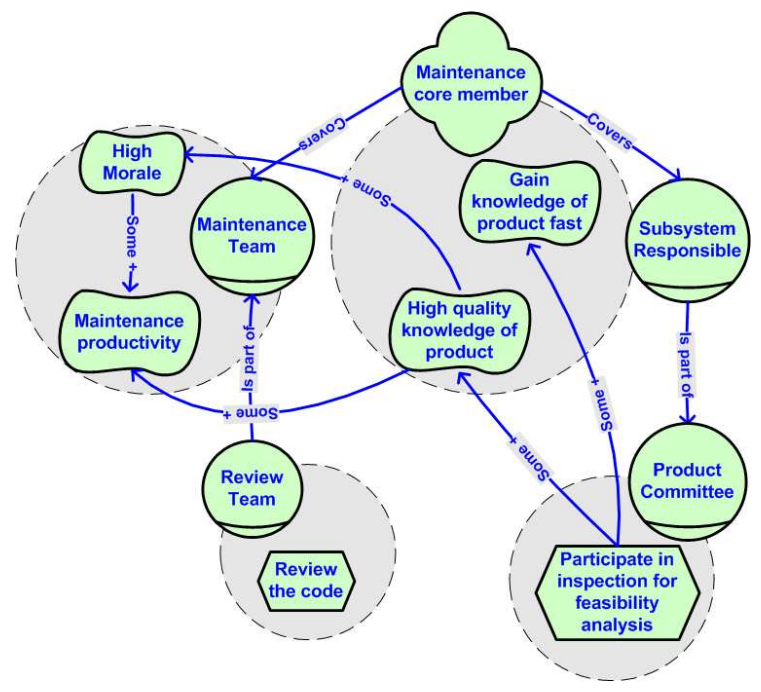

Fig. 11. A new model of the strategic relationships in the maintenance team and the impacts of the relationships on goals of team members. This model corresponds to the model in Fig. 7.

Properties of the Alternate Set of Models. The researchers tend to develop one large and comprehensive model rather than multiple simple models. These models contain more actors and complex relationships. The complexity of new models may stem from the fact that researchers have seen and analyzed the original models; therefore, they added more details to the original models.

While the title of roles in the original models express the tasks or goals of the roles, in the new models, tasks and goals of the roles and positions are $i^{*}$ elements inside the boundary of the actors. Adding this explicit structure increases expressiveness and understandability of the new models, while results in more complicated models. The new models developed by researchers also make use the full range of available i* modeling elements such as tasks and softgoals in addition to goals, and relationships such as contributions, decompositions, and means-end in addition to dependencies.

The models developed at Ericsson are simpler than the models developed by researchers in terms of number of elements, relationship between elements, and variety of $i^{*}$ elements used in the models. Simple models are better for understanding the problem for new users of the $\mathrm{i}^{*}$ models. In addition, simpler models, while conveying less information, are easier for communicating the information. On the other hand, simple models, which do not capture details of goals' refinement, task decompositions, and goal contributions, do not provide required basis for reasoning about the goal satisfaction and denial.

Goals in the Ericsson models are not related to any other subgoals or higher goals; therefore, if analysts wanted to evaluate satisfaction or denial of goals, they would have to make judgment based only on external evidence or own knowledge, 
not making use of the structure of the goal model. On the other hand, the goals in the models developed by the researchers are linked to subgoals, tasks, and higher (soft)goals. Therefore, the analysts can use the structure of the goal model to propagate satisfaction or denial values from leaf to root goals. In sum, the extensive and detailed models trade understandability and easier communication for the ability to perform reasoning using the model structure.

\section{Related Work}

Modeling and reasoning about enterprise knowledge can guide the organizational transformation. Enterprise Knowledge Modeling [6] is a collection of conceptual modeling techniques for describing different facets of the organizational domain. The relationship between EM and KM are studied in [7, where the authors concluded that EM and KM follow a merged future and both are required as the key contributors to decision making in an enterprise. Although knowledge creation activities are viewed as more important and more difficult to manage, effective knowledge reuse and knowledge transfer, as a pre-requirement for reuse, are more frequent organizational concerns [8].

Strohmaier et al. 9 argue that effectiveness of instruments used for knowledge transfer depend on the stakeholders that participate and share an interest in knowledge transfer and on their acceptance, motivation and goals. Therefore, goal-oriented EM approaches provide a suitable basis for modeling knowledge transfer instruments. For example, based on concerning stakeholders' willingness, goals, and conflicts, the contribution in 9] proposes an agent-oriented modeling approach, based on the $i^{*}$ Framework, for analyzing the effectiveness of knowledge transfer instruments, considering the goals from different interdependent stakeholders.

In another experience of using $\mathrm{i}^{*}$ for modeling knowledge transfer in a maintenance organization, Actor Dependency (AD) models of the $i^{*}$ Framework were used in an experience to model and analyze a large scale maintenance organization [10. The AD model was found to be very useful for capturing the important properties of the organizational context of the maintenance process, and aided in the understanding of the flaws found in this process.

\section{Conclusion, Limitations, and Future Work}

In this paper, we reported on the experience of applying the $i^{*}$ EM approach in the Ericsson Marconi SpA software maintenance organization to model and analyze their KM strategies. The modeling and analysis practices helped the practitioners to understand the significance and impact of the KM policies they applied, and enabled them to represent, capture, and analyze the strategic organizational relationships relevant to knowledge transfer and explain why they worked well with other teams.

Comparing the models developed at Ericsson with the models that the researchers developed, we concluded that the enterprise models developed in 
practice are relatively simpler and smaller. While, in this study, the researchers tend to develop one complicated model that illustrates the relation between smaller models, developing multiple models which each addresses one specific concern was more favorable in this experiment. This may stem from the lack of training or experience of the practitioner in developing complicated models. In addition, since the practitioner at Ericsson used the models as a communication and explanation tool, detailed models were not suitable for audiences which were not familiar with $i^{*}$ modeling. Although syntactical deviations from the $i^{*}$ notation in the models developed by the Ericsson practitioner are frequent, the models communicate the intuitions of the KM strategies and policies well. The syntax deviations provide further motivation for modifications to the $i^{*}$ syntax as suggested in [5].

These observations need to be considered in designing and revising EM languages such as $i^{*}$. This study shows the need for sub models as considered in EKD Frameworks [6] for reducing the complexity of the models. In order to develop sub models to divide the model into smaller consistent models which do not lose any information, slicing and grouping mechanisms are needed.

However, one major limitation of this study is drawing the conclusions based models developed in one single organization and by one modeler. A main threat to the validity of this study is the personal biases of the researchers. In this study, the developer of the alternate set of models and the researcher that analyzed the results are the same people. Therefore, the researcher may be biased for evaluating whether the new models are more understandable and expressive than original ones. In addition, this study and the conclusions about properties of enterprise models in real-world practices rely only on one experience and use of one specific modeling method in one specific experience. The conclusions drawn in this study may be valid only in a situation where the modeler is not trained for using a modeling and analysis method. Conclusions and hypothesis drawn in this study need to be evaluated by analyzing more practical models and also models developed using other EM methods.

\section{Acknowledgment}

Financial support from Natural Science and Engineering Research Council of Canada and Bell University Labs is gratefully acknowledged. The authors would like to thank Ericsson Software Research, at Marconi SpA, Italy, for providing the opportunity of the collaboration, and Jennifer Horkoff for useful discussions and comments.

\section{References}

1. Yu, E.S.K., Mylopoulos, J.: Understanding Why in Software Process Modelling, Analysis, and Design. In: Proc. of 16th ICSE, pp. 159-168 (1994)

2. Yu, E.: Modeling Strategic Relationships for Process Reengineering, Ph.D thesis, Department of Computer Science, University of Toronto, Canada (1995) 
3. Annosi, M.C., De Pascale, A., Gross, D., Yu, E.: Analyzing knowledge transfer in software maintenance organizations using an agent- and goal-oriented analysis technique- an experience report. In: Proc. of 3rd Int. i* Workshop, pp. 5-8 (2008)

4. i* Guidelines, version 3 (2007), http://istar.rwth-aachen.de/tiki-index.php?pageref id=67

5. Horkoff, J., Elahi, G., Yu, E., Abdulhadi, S.: Reflective Analysis of the Syntax and Semantics of the i* Framework. In: Second Workshop on Requirements, Intentions and Goals in Conceptual Modeling (RIGiM 2008) (to appear, 2008)

6. Bubenko, J.A., Persson, A., Stirna, J.: EKD - Enterprise Knowledge Development, User Guide (2001), http://www.dsv.su.se/ js/ekd_user_guide.html

7. Ted Goranson, H., Huhns, M.N., Nell, J.G., Panetto, H., Carbó, G.T., Wunram, M.: A Merged Future for Knowledge Management and Enterprise Modeling. In: ICEIMT, pp. 37-50 (2002)

8. Markus, M.L.: Towards a Theory of Knowledge Reuse: Types of Knowledge Reuse Situations and Factors in Reuse Success. Journal of Management Information Systems 18(1), 57-93 (2001)

9. Strohmaier, M., Yu, E., Horkoff, J., Aranda, J., Easterbrook, S.M.: Analyzing Knowledge Transfer Effectiveness - An Agent-Oriented Approach. In: Proc. of the 40th Hawaii Int. Conf. on System Sciences (2007)

10. Briand, L., Melo, W., Seaman, C., Basili, V.: Characterizing and Assessing a LargeScale Software Maintenance Organization. In: Proc. of the 17th Int. Conf. on Software Engineering, pp. 24-28 (1995) 